\title{
Républicanisme et libéralisme dans le moment révolutionnaire
}

Republicanism and Liberalism in the Revolutionary moment

Jean-Fabien Spitz

\section{(2) OpenEdition \\ 1 Journals}

\section{Édition électronique}

URL : https://journals.openedition.org/ahrf/11518

DOI : $10.4000 /$ ahrf.11518

ISSN : 1952-403X

Éditeur :

Armand Colin, Société des études robespierristes

\section{Édition imprimée}

Date de publication : 1 octobre 2009

Pagination : $19-45$

ISBN : 978-2-200-92560-4

ISSN : 0003-4436

\section{Référence électronique}

Jean-Fabien Spitz, «Républicanisme et libéralisme dans le moment révolutionnaire », Annales historiques de la Révolution française [En ligne], 358 | octobre-décembre 2009, mis en ligne le 01 octobre 2012, consulté le 22 avril 2022. URL : http://journals.openedition.org/ahrf/11518 ; DOI https://doi.org/10.4000/ahrf.11518 


\section{RÉPUBLICANISME ET LIBÉRALISME DANS LE MOMENT RÉVOLUTIONNAIRE ${ }^{1}$}

Jean-Fabien SPITZ

Cet article procède à une lecture critique de deux ouvrages récents parus aux États Unis qui, tous deux, se penchent du point de vue de I'histoire des idées sur la question du rapport entre langage républicain et langage libéral. Leur conclusion commune est que la tentative de réactualisation analytique du langage politique du républicanisme est un échec parce que, globalement, ce dernier

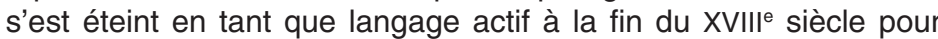
laisser la place à un langage libéral nettement distinct du républicanisme dont il a pris la place dans la mesure où il insiste fortement sur la préservation des activités privées, sur le caractère subordonné des activités civiques et sur une liberté essentiellement négative. L'article plaide au contraire pour l'idée que le langage républicain reste une ressource intellectuelle exploitable aujourd'hui et souligne que son originalité réside dans l'implication du concept d'égalité - égalité de moyens d'indépendance et de ressources de développement - dans le concept de liberté.

Mots-clés : république, républicanisme, libéralisme, liberté, égalité, langage politique.

Tout le monde admet aujourd'hui que le développement d'une philosophie politique républicaine est un des faits marquants de ces trente

(1) Le présent article est une étude critique des deux ouvrages suivants : Andreas KaLYvaS et Ira Katznelson, Liberal Beginnings. Making a Republic for the Moderns, Cambridge, Cambridge University Press, 2008 ; Andrew JAINCHILL, Reimagining Politics after the Terror : The Republican Origins of French Liberalism, Ithaca et Londres, Cornell University Press, 2008. Sur ce second ouvrage, $c f$. le compte rendu de Yannick Bosc, La Constitution de l'an III, un républicanisme classique (http://revolution-francaise.net/2008/09/06/258-constitution-an-III-republicanisme-classique). 
dernières années - l'ère post marxiste - dans le domaine de la théorie politique. En revanche, la question de savoir si le républicanisme constitue une véritable alternative sui generis au langage conceptuel de la tradition libérale demeure une vexata quaestio. Les principaux protagonistes du républicanisme contemporain croient bien entendu à la réalité de la différence et ont tenté de la préciser sur le plan analytique ${ }^{2}$; certains sont sceptiques et pensent qu'il existe une identité fondamentale de valeurs entre langage républicain et langage libéral, la divergence ne portant au fond que sur différents moyens de réaliser une aspiration commune à une forme de liberté essentiellement négative ${ }^{3}$; d'autres enfin pensent que les deux langages ont bien des origines distinctes, mais qu'ils sont si loin d'être mutuellement incompatibles entre eux qu'il conviendrait de plaider pour une théorie mixte qui emprunterait à la fois au marché et à la vertu ${ }^{4}$.

L'intérêt du livre d'Andreas Kalyvas et Ira Katznelson est de proposer de résoudre cette question sur un cas d'espèce en étudiant la théorie politique libérale in statu nascendi et en montrant comment elle s'est en réalité constituée à partir d'un langage républicain préexistant qui était sans aucune ambiguïté possible le langage politique dominant de l'opposition lors de la crise et de l'effondrement des monarchies à la fin du $\mathrm{XVIII}^{\mathrm{e}}$ siècle. La thèse de ce livre est donc que le langage libéral ne préexiste pas à cette crise en tant que langage pleinement constitué, mais qu'il émerge au moment où, confronté à la crise des monarchies, le langage républicain transmis par la tradition s'avère incapable de fonder un ordre politique stable susceptible de marier efficacement l'ordre à la liberté en repoussant simultanément les excès du despotisme et du démocratisme exacerbé qui auraient caractérisé les cités antiques. C'est donc à l'épreuve de la réalité - lorsqu'il a été pour ainsi dire sommé de présider à la naissance d'une réalité politique institutionnelle stable - que le langage oppositionnel républicain s'est profondément transformé pour devenir ce que nous reconnaissons aujourd'hui sous le nom de constitutionalisme libéral : «Le discours, les motivations et les concepts républi-

(2) Philip Pettit, Républicanisme, une théorie de la liberté et du gouvernement, Paris, Gallimard, 2000 .

(3) Alan Patten, «The Republican Critique of Liberalism », British Journal of Political Science, 26 (1996), p. 25-44.

(4) Richard DagGer, «Autonomy, Domination, and the Republican Challenge to Liberalism », dans John Christman et Joel Anderson (dir.), Autonomy and the Challenges to Liberalism, Cambridge, Cambridge University Press, 2005, p. 177-203; Richard DagGer, Civic Virtues. Rights, Citizenship, and Republican Liberalism, New York, Oxford University Press, 1997. 
cains n'ont pas été abandonnés - soutiennent Kalyvas et Katznelson - mais adaptés. Grâce à l'injection de significations, de justifications et d'arguments inédits dans des idées et des formes politiques préexistantes, on a façonné, à l'usage d'une république moderne, une doctrine dont le cœur est étonnamment libéral $»^{5}$.

L'approche analytique contemporaine engendrerait donc l'erreur historique consistant à affirmer que chacun des deux langages possède une généalogie qui lui est propre et que l'histoire des idées politiques à l'époque moderne est faite du dialogue et du débat entre deux approches qui sont et qui se savent mutuellement exclusives. Loin de posséder une généalogie distincte, le libéralisme s'est en réalité progressivement développé de l'intérieur du langage républicain et sous l'impact de la mise en lumière de son inadaptation ou de son inadéquation à la tâche à laquelle il était confronté : occuper, dans les conditions modernes, la place de la monarchie en tant que fondement intellectuel d'un ordre politique stable en faisant une place à ces deux exigences apparemment contradictoires que sont d'une part l'ordre et la stabilité, d'autre part la liberté et la souveraineté populaire qu'elle implique. Certes, cette transformation de l'intérieur suppose aussi l'apport d'éléments issus de traditions de pensée entièrement étrangères au républicanisme (par exemple la théorie des droits naturels, la tolérance et la prise en compte du pluralisme moral et religieux), sans que, toutefois, ces éléments extérieurs aient pu constituer un langage pleinement développé et politiquement utilisable comme outil d'opposition à la monarchie avant la seconde moitié du XVIII' siècle.

Mais outre l'erreur historique, l'approche analytique contemporaine engendre aussi l'erreur - bien plus importante - consistant à croire que le langage républicain demeure disponible en tant qu'alternative au libéralisme. La réalité est au contraire que, plus ce langage républicain s'est adapté pour devenir une « culture de gouvernement », plus il a perdu de sa pertinence et de sa viabilité en tant que langage distinct et plus il est devenu libéral. Au terme de ce mouvement - au premier tiers du $\mathrm{XIX}^{\mathrm{e}}$ siècle - il aurait donc purement et simplement disparu en tant que ressource intellectuelle autonome pour penser le problème d'une fondation institutionnelle de la liberté dans une société moderne et marchande.

(5) Andreas Kalyvas et Ira Katznelson, op. cit., p. 5 ; cf. ibid, p. 10 : « À mesure que les philosophes républicains s'efforçaient de rénover l'ancienne idée de la république pour l'adapter aux conditions modernes, et à mesure qu'ils luttaient par conséquent pour moderniser cette idée, ils ont inventé des idées et des institutions qui ont littéralement transformé le républicanisme classique pour en faire ce que nous connaissons maintenant sous le nom de libéralisme ». 
Toute tentation ou toute volonté d'y avoir recours pour critiquer ou mettre en cause la validité du mode de régulation libérale est dès lors une erreur fondamentale, car le langage libéral a conservé toute la part de l'héritage de son ancêtre qui était compatible avec la société moderne et permettait de la stabiliser (en particulier certains doutes sur les excès de l'individualisme), tandis que ce qu'il en a rejeté était en revanche profondément incompatible avec une telle société (en particulier le rejet du pluralisme et l'aspiration à l'unité abstraite). Tenter de l'utiliser aujourd'hui serait nier ce que les adeptes du langage républicain ont eux-mêmes reconnu à la fin du XVIII ${ }^{e}$ siècle, à savoir qu'il s'agit d'un langage incompatible avec la forme moderne de la société et incapable d'y fonder un ordre conjointement stable et libre.

Quelles sont les conditions modernes auxquelles le langage républicain a été requis de s'adapter? Quelles sont les transformations sociales qui ont exigé l'évolution du langage républicain et son aggiornamento ? La réponse n'est pas très difficile : la naissance des États centralisés de grande dimension, l'apparition de systèmes juridiques formalisés, le pluralisme moral et avant tout religieux au lendemain de la Réforme, la circulation accrue des marchandises et l'apparition d'une économie nouvelle donnant naissance à une nouvelle classe «bourgeoise » et exigeant une séparation plus étanche entre l'État et la sphère des activités privées. Dans ce contexte entièrement nouveau, aucun gouvernement stable ne peut exister s'il ne tient pas compte de la réalité et de la diversité des intérêts individuels, s'il ignore la nécessité d'obtenir le consentement au moins tacite des gouvernés, s'il n'admet pas que la dimension politique est dans une large mesure un instrument de protection d'activités qui en sont indépendantes, et s'il n'enregistre pas comme une donnée structurelle le scepticisme à l'égard de la capacité du politique à former des citoyens vertueux, toutes choses inaccessibles au langage républicain tel qu'il était transmis par la culture politique du milieu du XVIII ${ }^{\mathrm{e}}$ siècle.

Les auteurs étudiés par Kalyvas et Katznelson (Smith, Ferguson, Paine, Madison, De Staël et Constant) se sont tous préoccupés, implicitement ou explicitement d'actualiser le langage républicain afin de le mettre en mesure de tenir compte de ces impératifs et de pouvoir ainsi présider à la naissance d'une république moderne, mais jamais ils n'ont délibérément opté pour un langage libéral qui se serait trouvé déjà disponible comme alternative au vocabulaire du républicanisme classique. S'ils ont bien, avec d'autres, donné naissance à ce langage libéral, c'est à titre de résultat non recherché d'un effort de remaniement du langage dont ils avaient hérité et qui, au moment de combler le vide laissé par la disparition 
des monarchies, paraissait ne pas être adéquat à sa tâche historique. Mais précisément, le fait que le libéralisme soit né de cette mue et qu'il ne jouisse pas d'une généalogie entièrement indépendante interdit de le penser sous la forme caricaturale qui lui est donnée dans le cadre d'une approche purement analytique et détachée de toute considération touchant à l'histoire de ses «commencements ». On présente en effet ce langage sous la forme d'un système d'oppositions simples : la primauté du juste sur le bien, le recours à des procédures formelles et neutres et non plus à des valeurs morales substantielles, une vision strictement négative et non pas positive de la liberté, une accentuation de l'importance des personnes individuelles munies de droits et animées d'intérêts égoïstes aux dépens de la communauté, du bien public et des ressorts moraux de l'action.

Cette manière binaire de raisonner, qui affirme que le libéralisme est radicalement indifférent à chacun des seconds termes de ces alternatives, coupe le libéralisme de certaines de ses sources et façonne une théorie politique qui est bien trop étroite pour faire face aux problèmes de notre temps. Les créateurs du langage qui allait devenir celui du libéralisme constitutionnel refusaient précisément de penser dans des termes aussi tranchés : ils n'ont jamais abandonné le bien public ni l'idée de vie civique ; ils n'ont jamais glorifié de manière exclusive l'intérêt égoïste et personnel, ni refusé de considérer que les ressorts de l'action humaine pouvaient être essentiellement moraux. Tous ont certes pensé que les conditions nouvelles du monde moderne - en particulier le développement du capitalisme marchand, mais aussi la formation de sociétés pluralistes sur le plan religieux et sans doute aussi moral, ainsi que le développement de rapports civils beaucoup plus globalisés - exigeaient l'abandon des dogmes républicains les plus étroits et le recours à d'autres outils conceptuels pour négocier les tensions propres au monde moderne. Mais tous ont été formés dans le langage du républicanisme classique et tous sont demeurés sensibles aux thèmes auxquels il donnait un large écho : l'importance de la citoyenneté, l'irréductibilité de la société à un contrat entre personnes privées, la réalité des motivations morales et du bien commun, etc. Tous ont donc tenté de penser le caractère inédit des conditions d'institution de l'autorité politique dans le monde moderne mais aucun n'a voulu répudier le républicanisme classique dans son intégralité, car tous ont compris que les difficultés auxquelles les sociétés modernes sont inévitablement confrontées ne peuvent être résolues au moyen d'un langage partial et amputé, que celui-ci soit issu de la république classique ou des droits de l'individu. Ils se sont demandé, par exemple: dans quelle mesure l'inégalité engendrée par l'économie de 
marché est-elle compatible avec l'idée de souveraineté populaire ? Si le marché tend à priver le peuple de sa souveraineté et à la placer entre les mains des détenteurs de la richesse, est-il possible de faire obstacle à cette dérive et, si oui, par quels moyens ? Comment concilier l'appétit de pouvoir des États face à la concurrence de leurs rivaux et l'exigence de la poursuite de la paix par le moyen du droit et des institutions internationales ? Comment résoudre le dilemme de la liberté et de la sécurité ? Comment définir de nouveaux critères d'appartenance à une communauté politique et concilier ainsi les impératifs universalistes avec la reconnaissance des singularités religieuses et morales ? Autant de questions dont, manifestement, la réponse ne peut pas plus venir du seul langage des droits que du seul vocabulaire de la république classique. Comment, en effet, penser le marché à l'aide du seul concept de vertu ? Mais comment, inversement, penser sa limitation sans ce concept, puisque toute restriction de la liberté d'acquérir et de posséder exige une attention au droit de l'autre, donc une forme de vertu et une forme d'adhésion au bien commun? Comment limiter la puissance de l'État sans le concept formel du droit? Mais comment faire une place à la nécessité de la puissance sans un concept de patrie ? etc. Ce sont précisément ces tensions qui ont poussé ces théoriciens à adapter le langage dont ils disposaient pour faire place aux instruments conceptuels qui leur permettaient d'affronter les défis inédits du monde moderne - ceux que la cité classique ne pouvait pas poser dans les mêmes termes puisqu'elle ignorait tout de la société civile, de l'État moderne, des droits, du contrat, du marché, et du pluralisme moral. Dans le cours de cette adaptation, ils ont créé un mixte entre des éléments qui peuvent aujourd'hui nous paraître antithétiques, mais ce mixte est sans doute un équilibre entre les exigences contradictoires dont il fallait tenir compte dans l'entreprise de stabilisation d'un ordre politique au moment de la chute des monarchies : liberté et autorité, marché et vertu, commerce et civisme, individu et communauté, intérêt et devoir, etc.

Toute la question est de déterminer jusqu'où va le processus de remise en question et d'adaptation du langage républicain classique. Est-ce qu'il inclut, en particulier, la récusation de l'idée que l'autonomie du citoyen requiert des bases matérielles qui, à leur tour, exigent une limitation du droit de propriété et une attention scrupuleuse à la manière dont celle-ci est distribuée ? Si la réponse à cette question est « oui » et si le droit illimité et absolu de propriété fait nécessairement partie de l'outillage conceptuel des sociétés modernes, il est vrai que le républicanisme a disparu en tant que langage autonome. Mais si la nécessaire 
adaptation du langage de la république classique ne s'étend pas nécessairement jusqu'à la validation d'un tel concept absolutiste du droit de propriété, il y a encore place pour une réflexion sur la manière dont une société d'individus peut limiter le marché pour faire une place à la liberté égale de l'ensemble de ses membres ${ }^{6}$.

Par exemple, il est certain que, pour Adam Smith, le libre marché est un puissant facteur de liberté qui brise les hiérarchies traditionnelles, ainsi que tous les mécanismes d'exclusion et de privilège. À la différence des autres idéologies économiques de l'époque, comme le mercantilisme, il est fondé sur la libre expression des préférences individuelles et, au lieu de chercher à changer ou à réprimer la nature humaine de manière à la faire coïncider avec un ensemble abstrait de valeurs morales, il en accepte les tendances telles qu'elles sont et cherche à se rendre attentif à leurs caractéristiques.

Il est tout aussi clair que Smith développe une théorie de l'intégration sociale par la complémentarité des fonctions dans le contexte de la division du travail et non plus par un ensemble de valeurs morales partagées ou par une forme d'homogénéité sociale. Ces deux idées - vertu, morale partagée - étaient des caractères spécifiques du républicanisme classique, mais elles sont entièrement privées de validité dans des sociétés devenues pluralistes et composées d'individus essentiellement différents en raison de la complémentarité et de la spécificité de leurs fonctions.

On pourrait encore souligner à quel point Smith est convaincu que, dans les conditions de l'économie moderne, il devient possible aux hommes de vivre ensemble en allégeant au maximum le poids et la place de l'autorité collective car, même s'il génère des tensions et des conflits, le mode de production marchand tend à produire de la stabilité et jouit, avec le marché, d'un mécanisme qui équilibre et ajuste constamment les uns aux autres les composantes sociales et les intérêts en présence.

De tels concepts sont indiscutablement libéraux mais, comme cela est désormais admis par tous les chercheurs, ils n'épuisent pas le contenu de la philosophie sociale de l'auteur de La richesse des nations. Même si les tentatives pour dresser le portrait d'un Smith humaniste civique sont

(6) Rappelons que c'est l'hypothèse explorée par la Théorie de la justice de John Rawls, qui refuse d'inscrire le droit de propriété au nombre des libertés de base garanties par le premier principe de la justice comme équité et qui ne voit donc aucune contradiction dans la volonté d'établir de manière indépendante - sans l'appui d'un droit absolu de propriété - l'ensemble des libertés fondamentales qui définissent un régime de liberté constitutionnelle ( $c f$. John Rawls Théorie de la justice, Paris, Seuil, 1989, § 43). 
dépourvues de pertinence en tant que modèles globaux ${ }^{7}$, elles saisissent quelque chose qui, dans sa pensée, paraît irréductible à la forme caricaturale de libéralisme qu'on lui a si souvent attribuée. Smith suggère par exemple que la poursuite de l'intérêt matériel n'est pas nécessairement une donnée fondamentale de la nature humaine et que ce désir pourrait n'être qu'une forme dérivée d'une passion plus noble et plus fondamentale : le désir d'estime et le souci d'éviter la honte. Tout en exploitant cette ressource du vocabulaire classique, Smith la reformule cependant car, là où les anciens affirmaient que le désir d'estime conduit à des hauts faits dans l'exercice des fonctions publiques, civiles ou militaires, il affirme quant à lui qu'il prend la forme d'un désir d'enrichissement et de sécurité par la médiation de la paix et de la sécurité. Les hommes ne sont donc, à ses yeux, ni des atomes isolés ni le produit de sociétés réifiées ou de normes abstraites, mais ils sont au contraire constamment engagés dans des relations et dans des réseaux au sein desquels ils sont portés à adopter le point de vue des tiers. Il s'agit là d'un mécanisme qui amène chacun à estimer sa propre valeur dans le regard et l'approbation des autres, et qui conduit les individus à élargir leur propre univers mental, à s'insérer dans des réseaux sociaux plus vastes, et à négocier ainsi la qualité et le contenu de l'approbation mutuelle. Dans ces conditions, il ne peut exister de moi stable en dehors du contexte social d'une perception mutuelle, et la capacité que nous avons de nous former une personnalité éthique cohérente est directement liée à la forme et à l'ampleur de la structure plus vaste de rapports interpersonnels au sein de laquelle nous nous insérons ${ }^{8}$.

Certes, les anciens moyens de reconnaissance - la compétition politique et la guerre - sont devenus obsolètes dans les États modernes de grande dimension fondés sur le capitalisme marchand. La lutte pour la reconnaissance se déplace donc du terrain politique et militaire au terrain éthique, dans un effort de chacun pour se gagner l'approbation des autres au sein de la société civile et non plus sur la place publique. La rhétorique, l'art de persuader les autres, demeure le principal outil de fabrication de la reconnaissance, mais dans un autre domaine : elle enseigne désormais à chacun, dans le tissu des relations privées, comment manifester les

(7) Donald Winch, Adam Smith's Politics, An Essay in Historiographic Revision, Cambridge, Cambridge University Press, 1978.

(8) Andreas Kalyvas et Ira Katznelson, op. cit., p. 32 : «En faisant du désir d'estime le ressort principal, Smith combine le langage des droits naturels et l'idée d'une éthique située, au moment même où le concept d'une telle éthique semble mise en cause par les développements économiques propres aux sociétés modernes ». 
sentiments qui lui attirent la sympathie des autres et à se présenter soimême comme un être digne de reconnaissance et d'estime.

Le marché est au demeurant le lieu privilégié de cette persuasion car chacun, en tentant de vendre, tente aussi de persuader les autres de se ranger à son avis ; réussir à vendre, c'est donc être reconnu positivement, c'est être approuvé et voir notre activité frappée du sceau de l'utilité sociale. La quête de l'approbation par les autres demeure le ressort fondamental mais le langage des affaires et du commerce est maintenant devenu le principal moyen de la lutte pour la reconnaissance. Ce genre de persuasion qui se pratique sur le marché, ainsi que le genre de lutte pour la reconnaissance qui s'y livre, sont bien plus en accord avec les conditions modernes que la lutte politique et militaire de type agonistique à laquelle se livraient les anciens dans une société pauvre ${ }^{9}$. Le fondement descriptif et normatif de la théorie de Smith n'est donc pas une pulsion marchande fondamentale. Bien au contraire, c'est la persistante lutte pour la reconnaissance et la recherche de l'approbation sociale qui expliquent le désir des individus modernes d'accumuler de la richesse. Désormais, c'est la richesse qui attire la reconnaissance et l'autorité ; la politique agonistique des anciens s'est transformée en une agonistique économique. La richesse attire sur celui qui la possède l'attention du monde, le marché élève celui qui y réussit au rang d'un homme de mérite et de distinction ${ }^{10}$.

En développant une théorie de ce genre, on peut dire que Smith brouille les frontières entre des théories de la sociabilité naturelle d'une part, et de l'autre l'idée que toutes les relations sociales seraient purement intéressées ; il n'adhère ni au sens moral de ses prédécesseurs ni à l'égoïsme de Mandeville, mais il crée un mixte des deux, une sorte d'équilibre entre amour de soi et bienveillance, entre individu et communauté, entre le juste et le bien, entre la moralité formelle et l'éthique substantielle. Smith présenterait donc une solution alternative à Kant (la moralité purement formelle des droits) d'une part, et à l'utilitarisme de l'autre. Il aurait enrichi le cœur de la moralité séculière grâce à une théorie centrée sur les relations affectives réciproques et sur des formes substantielles de communication interpersonnelle qui transcendent la simple rationalité abs-

(9) Ibid., p. 39-40 : «Smith a perçu avec acuité que, bien que les hommes modernes ne luttent pas pour l'excellence publique au sein de l'arène politique, et bien qu'ils ne recherchent pas l'approbation ouvertement devant leurs concitoyens, ils n'en continuent pas moins d'aspirer à la reconnaissance de leurs pairs mais, désormais, ils la recherchent par des moyens économiques; l'échange marchand est non pas simplement un moyen utilitaire mais un vecteur de reconnaissance $»$.

(10) Ibid., p. 43. 
traite et les considérations utilitaires ou purement contractuelles sur les relations d'échange entre acteurs économiques intéressés.

Kalyvas et Katznelson étudient de la même manière le retravail que Paine et Madison font subir au vocabulaire de la république classique pour le rendre compatible avec le pluralisme et la liberté moderne en y injectant la théorie de la représentation, les droits naturels et la liberté de conscience. Un tel retravail consiste bien entendu à chercher une reformulation politique capable d'associer - c'est le sens de la constitution des États-Unis - la liberté et la stabilité, la souveraineté du peuple et le gouvernement par l'élite représentative. Il s'agit bien d'une entreprise de défense de la forme républicaine, destinée à montrer qu'elle est compatible avec un État pluraliste de grande dimension et qu'elle n'entraîne pas nécessairement - à condition d'être fédérale et représentative - les dérives inhérentes au gouvernement démocratique : l'intolérance et l'oppression des minorités. Mais cette reformulation - qui se traduit par l'apport d'éléments étrangers au républicanisme classique, en particulier le refus de la religion civique et de la toute puissance du peuple ainsi que l'affirmation des droits individuels - ne signifie pas que le gouvernement soit devenu le simple instrument des passions individuelles car Paine et Madison insistent tous deux sur l'idée que cette république inédite est aussi le seul moyen qui permette à un peuple de grande dimension d'agir collectivement pour maîtriser son destin : il s'agit bel et bien d'augmenter le pouvoir du peuple sur lui-même et non pas de le réduire ! Et si la république, attentive à la diversité, n'est pas la communauté fermée et sectaire de l'antiquité, elle n'est pas non plus dominée par l'égoïsme des passions car et Paine et Madison sont sensibles à une anthropologie républicaine qui souligne la force et l'importance des affections sociales.

Mais, paradoxalement, c'est chez deux auteurs français $-\mathrm{M}^{\mathrm{me}} \mathrm{de}$ Staël et Benjamin Constant - que Kalyvas et Katznelson voient s'accomplir, sous l'impact des aberrations auxquelles donne lieu, dans la révolution française, la mise en œuvre d'un vocabulaire politique républicain non épuré, la conversion la plus décidée à un langage qui au bout du compte et après des hésitations, ne conserve pratiquement plus rien du langage classique dont il est la reformulation et dont il est issu.

$\mathrm{M}^{\mathrm{me}}$ de Staël et Benjamin Constant ont tous deux constaté que les idées républicaines, telles qu'elles sont transmises au moment de la révolution, sont incapables de frayer une voie moyenne entre le despotisme monarchique et le despotisme démocratique ou jacobin. Par des voies différentes, ils sont tous deux conscients de la nécessité de redéfinir le corpus des idées républicaines pour lui permettre de servir de socle à une 
liberté institutionnelle, et c'est sous l'impact de cette nécessité qu'ils deviennent libéraux. Selon Kalyvas et Katznelson, Constant va même au-delà de cette évolution vers un libéralisme des droits puisqu'il prend conscience de l'incapacité du vocabulaire individualiste et libéral à fonder une communauté suffisamment «épaisse » pour être solide et qu'il sent la nécessité d'y injecter des idées issues de ce que nos auteurs appellent la tradition : un pouvoir préservateur héréditaire et des idées morales partagées.

Dans leurs premiers textes, $\mathrm{M}^{\mathrm{me}}$ de Staël et Constant sont attachés à ce que l'on pourrait appeler une évolution modérée du vocabulaire républicain, dont l'objet serait essentiellement de parer aux dérives de l'élément démocratique et à son incapacité à se cantonner à une forme de légalité stable et constitutionnalisée. À leurs yeux, la démocratie possède en effet un pouvoir destructeur qui a démontré son efficacité dans la lutte contre le principe autocratique mais elle est incapable de donner naissance à un ordre juridique stable parce qu'elle est habitée à l'excès par l'idée que la volonté du peuple peut tout et qu'elle ne peut ni ne doit être assujettie à aucune forme ni à aucune limite. Pour faire obstacle à l'anarchie et à la tyrannie de la majorité, il suffirait cependant d'une constitution représentative qui viendrait remédier à l'incapacité du régime démocratique à se stabiliser par l'injection d'un élément aristocratique. La modernisation de l'idée républicaine se réduirait donc ici à une tactique destinée à guérir la démocratie de ses propres excès par l'inoculation d'éléments contraires à son principe, sans qu'il soit nécessaire de mettre l'accent sur les thèmes proprement libéraux de la garantie des droits individuels, de l'indépendance du judiciaire, de la suprématie du droit, et du respect scrupuleux des formes juridiques établies. $\mathrm{M}^{\mathrm{me}}$ de Staël répudie ainsi la monarchie à l'anglaise en soutenant que l'élément monarchique est par principe incompatible avec la liberté et la souveraineté du peuple.

Cette constitution « républicaine » modernisée demeure donc fort peu libérale ; pas ou peu de véritable séparation des pouvoirs, pas ou peu de respect du pluralisme religieux, une certaine tendance à considérer la religion civique comme une condition nécessaire de l'unité morale et sociale, un appel récurrent au patriotisme, une place faite au pouvoir d'exception indispensable pour garantir la forme même de la souveraineté populaire, et une dévotion réaffirmée au bien commun. En outre, les droits personnels y sont nettement conçus comme inférieurs aux droits politiques, vraie garantie de la liberté contre le principe despotique. La liberté individuelle, en ce sens, n'est au fond que la garantie de la possession des droits politiques qui demeure le point essentiel, et la protection de l'auto- 
nomie privée n'est justifiée que par le souci de protéger l'autonomie publique. Au demeurant, dit $\mathrm{M}^{\mathrm{me}}$ de Staël, chacun sait que la liberté civile est accessible sous un monarque absolu, alors que les droits politiques ne peuvent être garantis que par une constitution excluant tout principe autocratique ou héréditaire.

Mais en 1810, puis en 1818, le ton change et le souci de la souveraineté du peuple s'efface devant celui des libertés civiles. C'est le tournant libéral au cours duquel $\mathrm{M}^{\mathrm{me}}$ de Staël réinterprète l'histoire occidentale comme un désir de liberté civile sous la forme d'une indépendance personnelle par rapport à l'autorité collective ; elle voit désormais le moment de l'assemblée constituante et de la déclaration des droits de l'homme et du citoyen comme le moment vraiment pur de la révolution libérale qui donne naissance aux éléments d'une authentique liberté civile en termes de sécurité individuelle et de protection contre l'arbitraire de l'État et de la majorité, désormais conçu comme le principal danger qui menace la liberté des modernes. Ce tournant la détermine à rejeter le patriotisme comme une forme dangereuse d'archaïsme et à nier tout intérêt national ou commun qui pourrait, dans des circonstances exceptionnelles, être supérieur aux libertés individuelles fondamentales. Le langage politique du libéralisme tend alors vers sa complétude : droits individuels inaliénables constitutionnalisés et protégés contre la volonté majoritaire ; approbation du gouvernement anglais qui protège la sphère individuelle d'action et garantit l'habeas corpus ; inconditionnalité de la liberté de la presse ; répudiation de l'idée de religion d'État, et approbation de la liberté religieuse et de la tolérance ; affirmation du caractère purement privé de la religion; prééminence des droits civils sur les droits politiques ; réfutation de l'idée que l'égalité serait une composante essentielle de la liberté ; récusation de toute idée selon laquelle la cité devrait être moralement homogène au profit d'une logique du pluralisme des intérêts ; nécessité d'établir les institutions d'une manière telle que chaque individu ait un intérêt personnel à les soutenir; et enfin adhésion sans faille au principe de la juridicité formelle et au principe de la séparation des pouvoirs.

La raison de ce tournant libéral est aisée à comprendre : la théorie républicaine est incapable de tenir le centre entre démocratie et despotisme, et elle incline de manière excessive vers la démocratie pure, donc vers la tyrannie de la majorité ; en d'autres termes, elle ne permet tout simplement pas de garantir la liberté individuelle, et l'injection de mécanismes représentatifs dans la constitution républicaine ne suffit pas pour cela sans l'injection additionnelle de l'idée de droits inaliénables. En outre $\mathrm{M}^{\mathrm{me}}$ de Staël remarque que la république fait appel à la vertu, une 
denrée introuvable dans l'époque modern et dont le défaut implique immanquablement, comme l'a démontré le cours de la révolution, le recours à la coercition à titre de substitut. En revanche, le libéralisme est capable de se passer de la vertu pour garantir la liberté car il n'a pas besoin de l'intervention active du peuple et ne se trouve pas acculé à recourir à la contrainte pour en pallier le manque ${ }^{11}$.

Dès lors, le républicanisme n'est plus la solution au problème de la liberté mais au contraire ce qui y fait obstacle. $\mathrm{M}^{\mathrm{me}}$ de Staël en arrive à la conclusion qu'il y a un lien intrinsèque entre le républicanisme et la Terreur, et que le jacobinisme est le fils naturel du langage classique qui entraîne nécessairement une tentation extrémiste, un mépris des garanties, des limitations et des contraintes imposées à l'autorité centrale de l'État. Il s'agit de doctrines destructrices qui sont incapables de construire un ordre politique stabilisé.

Benjamin Constant partage avec $\mathrm{M}^{\mathrm{me}}$ de Staël cette évolution d'une transformation limitée du langage républicain par l'injection d'éléments de représentation de nature aristocratique au tournant libéral qui lui fait comprendre que l'État est bien le lieu majeur de la domination et l'ennemi essentiel de la liberté dans les sociétés modernes. Si, dans le Fragment d'un ouvrage abandonné sur la possibilité d'une constitution républicaine pour un grand pays ${ }^{12}$, il accentue encore l'égalité et l'aspect démocratique des institutions aux dépens des droits individuels et du libéralisme économique, il change manifestement d'orientation dans les Principes de politique applicables à tous les gouvernements de 1802-1806, pour formuler à son tour un impeccable programme libéral : respect des formes judiciaires, minoration du caractère démocratique du pouvoir, importance croissante de l'idée que la figure emblématique de l'arbitraire politique est un pouvoir démocratique envahissant les droits des individus et en particulier le droit de propriété ; appel à la neutralité morale de l'État.

Mais, au-delà de cet incontestable « tournant libéral », Constant, dans son dernier ouvrage ( les Principes de politique applicables à tous les gouvernements représentatifs de 1815) se rend compte que les principes purement libéraux sont insuffisants pour stabiliser le lien social, que les sociétés modernes sont guettées par l'excès de privatisation et que le régime économique moderne pourrait bien engendrer de nouvelles formes d'oligarchie ; il est conscient aussi que le caractère formel des droits risque d'engendrer une passivité politique, permettant ainsi à une minorité

(11) Andreas Kalyvas et Ira Katznelson, op. cit., p. 142.

(12) Édition Henri Grange, Paris, Aubier, 1991. 
agissante de mettre la main sur le pouvoir au détriment du principe de la légitimité démocratique et de l'autonomie politique des citoyens.

Mais au lieu de revenir à une forme d'esprit public d'inspiration républicaine, Constant se tourne vers l'idée d'une monarchie constitutionnelle. Un régime exclusivement centré sur une représentation parlementaire, dit-il maintenant, risquerait non seulement de devenir oppressif (les élites élues s'annexant le pouvoir) mais serait aussi privé d'un principe efficace d'exécution : ce serait un régime indécis, incapable d'agir, amené à se paralyser lui-même par ses propres divisions ${ }^{13}$. Un roi est donc nécessaire à la fois pour l'énergie mais aussi pour la neutralité puisqu'il n'est pas partie prenante des conflits d'intérêt. Il faut un pouvoir neutre qui soit à distance par principe de tous les conflits de partis et d'intérêts, et il faut en outre un second élément issu de la tradition : puisque l'intérêt particulier qui domine l'âge moderne tend à isoler chaque individu de ses concitoyens, le lien social ne peut se solidifier efficacement sans le recours à des croyances morales partagées. Ces éléments traditionnels, le roi et la moralité commune - Constant va même jusqu'à parler d'enracinement, de liens communuataires forts - viennent combler les manques d'une conception trop formellement libérale, et entretiennent parfois de curieux rapports de voisinage avec des éléments directement issus du langage de la république archaïque comme l'éloge du patriotisme et de l'engagement citoyen ${ }^{14}$.

Trois couches donc : un pouvoir populaire et républicain qui fait la légitimité, mais circonscrit par des droits individuels, eux-mêmes encadrés dans leur sécheresse et leur abstraction par des éléments issus de la tradition $^{15}$. Trois principes de légitimité : le peuple, les droits, la tradition, le tout étant gouverné par la recherche d'un ordre à la fois libre et stable. C'est bien l'incapacité du républicanisme classique ( qui ne possède ni les droits ni l'appel à la tradition ) à supplanter ou à remplacer la monarchie dans la fondation d'un ordre politique à la fois libre et stable qui a déterminé Constant à s'en éloigner et à chercher une autre solution de remplacement à la monarchie : il la trouve dans un libéralisme qui n'est plus républicain mais qui n'est pas non plus intégralement libéral puisqu'il n'est ni utilitariste, ni contractualiste, ni attaché à l'idée que les seuls liens entre les individus sont des liens d'intérêt. La tradition vient colmater les brèches d'un libéralisme trop décommunautarisé et trop individualiste.

(13) Andreas Kalyvas et Ira Katznelson, op. cit., p. 169.

(14) Rappelons que le texte sur la liberté des anciens et sur la liberté des modernes se termine sur un vibrant appel à combiner les deux formes de la liberté.

(15) Andreas Kalyvas et Ira Katznelson, op. cit., p. 174. 
C'est ce que Kalyvas et Katznelson appellent un «libéralisme immanent » : des droits enserrés dans un terrain historiquement déterminé et entretenu comme tel, des traditions, des éléments stables issus de l'histoire, des croyances et des moralités communes. La théorie des droits finalement proposée par Constant ne serait donc pas une théorie purement formelle, contractualiste ou jusnaturaliste, mais elle serait contextualisée, inscrite dans l'histoire, particularisée, centrée sur une patrie, et retenant ainsi quelque chose du langage qu'elle avait commencé par répudier comme incompatible avec la liberté moderne.

L'idée que le langage proprement libéral de la protection des droits individuels ne préexiste pas réellement à la révolution et que le langage de la république classique demeure, jusqu'à Thermidor et par-dessus l'épisode de la Terreur, une matrice commune qui ne se convertit que très lentement aux idées centrales du libéralisme est également au cœur de l'ouvrage d'Andrew Jainchill.

Selon ce dernier, on a souvent considéré que, en France, le républicanisme classique cristallisé dans la pensée de Rousseau était venu culminer dans le langage politique de la Terreur jacobine ${ }^{16}$. Une conséquence de cette intuition est que lorsque, dans les années 1970, un certain nombre d'intellectuels ont pris un «tournant anti totalitaire », ils ont proposé une réévaluation de la tradition républicaine à laquelle la gauche s'identifiait et conclu qu'il convenait de l'abandonner au profit d'un retour aux sources du libéralisme français. Constant, Guizot et Tocqueville devaient ainsi servir d'antidote à une forme de républicanisme marquée par les idées de vertu et de bien public, l'hyperdémocratisme, le culte des masses, et par la tentation autoritaire du façonnement des mœurs patriotiques et de l'imposition des valeurs partagées. Ce libéralisme, les adeptes du tournant anti-autoritaire le voient poindre dès les lendemains de Thermidor, qui aurait représenté, selon l'expression de François Furet, le passage d'un type de pouvoir à un autre, infiniment moins anti pluraliste et plus accueillant aux idées libérales de droit individuel et d'autonomie de la société civile ${ }^{17}$. Suivant en cela les conclusions de Bronislaw Baczko ${ }^{18}$, A. Jainchill souhaite au contraire contester cette thèse qui fait de Ther-

(16) Par exemple Keith Michael BAKER, « Transformations of Classical Republicanism in Eighteenth Century France », Journal of Modern History, 73, 2001, p. 35-56.

(17) Cf. Helen Rosenblatt, « Why Constant ? A critical overview of the Constant revival », Modern Intellectual History, 1, 2004, p. 439-453 ; Michael S. CHRISTOFFERson, French Intellectuals Against the Left : the Antitotalitarian Moment of the 1970s, New York, Berghahn Books, 2004.

(18) Bronislaw BACzKo, Comment sortir de la Terreur. Thermidor et la Révolution, Paris, Gallimard, 1989. 
midor un véritable changement d'époque et une entrée - même timide dans l'univers du libéralisme. Pour lui le langage politique des années 1794-1799 demeure fortement marqué par le républicanisme classique, réalité difficile à percevoir dans la mesure où l'on a caricaturé cette forme de pensée en la confondant avec les errements de la période Jacobine. Or cette dernière se démarque en fait assez nettement du langage du républicanisme classique : elle a un aspect millénariste entièrement étranger à un langage qui insiste au contraire sur le fait que le temps historique est cyclique et voué à une forme de dégénérescence, que la république n'est pas un achèvement triomphal du bien, mais une réalité précaire, inscrite dans le temps par l'œuvre des hommes ( et non d'une transcendance mystique comme le Peuple ), exposée à la corruption, ancrée dans l'expérience et le tâtonnement bien plus que dans les vérités abstraites de la philosophie des Lumières. La république classique ne croit pas aux droits abstraits donnés par la nature mais aux droits concrets et patiemment égalisés à force d'expérience, incarnés et rendus réels par une forme institutionnelle mixte et savamment dosée; elle ne croit pas à l'égalité naturelle, mais à une égalité civile fortement tempérée par le rôle des élites incarnant une prudence qui fait nécessairement défaut au peuple ; elle ne croit pas à la vertu innée des masses mais aux mœurs issues d'une longue pratique, à la formation d'une élite de citoyens éclairés ; elle ne croit pas à la démocratie ni au volontarisme du Peuple abstrait, mais à la rationalité des citoyens propriétaires indépendants, mûrs, conscients de l'indissociabilité de leur intérêt particulier et de celui de la patrie.

Il y a donc un écart significatif entre République et Jacobinisme, car la première prend l'histoire pour code et tente d'instaurer, à l'intérieur du temps des hommes, une forme politique capable de limiter la dépendance et les phénomènes de pouvoir. À l'inverse de la fuite en avant jacobine vers une forme de paroxysme libérateur, la république est à la recherche des mécanismes institutionnels d'équilibre et de compensation qui permettront de stabiliser la liberté et de faire vivre un régime durable capable de maîtriser les passions dissociatrices et les formes de corruption, d'inégalité et de pouvoir, qui entraînent la mort de la chose publique et la désaffection des citoyens envers leurs institutions. Contrairement à ce que Jainchill semble suggérer, ce langage républicain avait existé en France avant 1789, et il est donc abusif de prétendre que la France «n'a pleinement participé au Moment Machiavélien de l'aire euro-atlantique » qu'après la chute de Robespierre. L'essentiel de la thèse défendue par Jainchill n'est cependant pas là car son intuition centrale est que les Thermidoriens sont plus républicains que libéraux et que leur rejet de la Terreur 
s'appuie plus sur l'idée que celle-ci a trahi la prudence et la recherche de stabilité équilibrée qui caractérisent la république que sur l'idée qu'elle a porté atteinte au pluralisme des intérêts, à l'indépendance de la société et au respect des droits individuels. C'est sans doute en raison de ce que le langage du républicanisme classique est devenu par la suite - il s'est mué en une variante très spécifique du libéralisme - que nous avons du mal à comprendre une réalité pourtant assez simple : ce langage était, avant 1789 , l'outil essentiel de la critique de la monarchie absolue et des propositions de reconstruction d'un ordre politique stable: souveraineté du peuple entendue comme l'ensemble des citoyens éclairés, indépendants et ayant un intérêt à la stabilité de la loi, équilibre des pouvoirs, liberté comme contrôle des dominations privées et des privilèges ; la déclaration des droits de l'homme et du citoyen elle-même porte la marque de cette prééminence des droits induits par la loi sur les droits de la nature et témoigne du fait que le langage libéral se trouve alors dans un état largement inchoatif. L'emballement révolutionnaire a certes provoqué une radicalisation du langage initial, un appel aux vérités abstraites et à l'idée d'égalité absolue, une injection d'aspects " chiliastiques » et de formes de transcendance que le républicanisme initial ne comportait pas, et ce sont ces transformations à chaud qui sont brutalement stoppées avec Thermidor au profit, non pas d'un libéralisme qui n'existe pas encore en tant que langage constitué où il serait possible de puiser les éléments d'un ordre politique stable comme solution de rechange tant à la monarchie qu'au jacobinisme, mais d'une réactualisation du langage républicain classique. Le libéralisme français, celui de Constant et de $\mathrm{M}^{\mathrm{me}}$ de Staël en particulier, est issu d'une mue très lente de ce républicanisme remis à l'ordre du jour après Thermidor, et il en conserve une empreinte qui lui donne une tonalité particulière. Telle est du moins la thèse défendue par Andrew Jainchill.

En quoi la réflexion politique des Thermidoriens est-elle marquée par le républicanisme classique?

Tout d'abord parce qu'elle cherche explicitement la stabilité à l'intérieur du temps des institutions, c'est-à-dire d'un temps spécifiquement humain. Ensuite, parce qu'elle cherche cette stabilité grâce à une forme de mixité et d'équilibre - même si la constitution de l'an III y échoue puisqu'elle sépare les pouvoirs au lieu de les partager - en recourant au bicamérisme et même à l'idée spécifiquement républicaine selon laquelle il convient de confier la délibération à l'une des assemblées et la décision à l'autre. Les thermidoriens ne pensent pas non plus que la volonté collective de la masse soit le dernier mot de la politique et ils font 
plutôt confiance à une élite de citoyens éclairés, vertueux, indépendants et conscients de leurs intérêts. La démocratie extrême n'est pas plus leur mot d'ordre que la participation de tous à la chose publique et, en cela, ils sont véritablement les héritiers d'un langage politique qui n'a jamais confondu république et démocratie et toujours soutenu que la volonté populaire devait être - pour le moins - équilibrée par la sagesse des élites, par leur capacité à percevoir les conséquences, à mesurer les enjeux, à prévenir les maux. L'imposition d'un cens électoral et le mécanisme de sélection des représentants - ainsi que le projet consistant à exiger que, à terme, tout électeur sache lire et écrire - traduisent ce souci d'assurer la prééminence des hommes les plus éclairés et les plus capables de prendre à cœur les intérêts de la patrie ; ces mécanisme suggèrent aussi, en ne retenant que la propriété foncière et non la propriété industrielle et commerciale dans le calcul du cens, que les thermidoriens demeurent sous l'influence de l'idée que le citoyen ne peut opiner de manière rationnelle s'il ne dispose pas des fondements d'une indépendance et d'une personnalité stable que la terre seule peut lui donner. A. Jainchill souligne également à quel point le Directoire s'est immédiatement préoccupé de la formation de cette personnalité stable chez les élites citoyennes en fondant à leur intention - et non à celle des masses populaires - une école centrale par département où il s'agissait autant d'éduquer le citoyen aux mœurs républicaines que de l'instruire.

L'approche de la question des droits est également révélatrice du réservoir auquel les thermidoriens viennent alimenter leurs conceptions politiques : il s'agit moins de protéger un pluralisme et une diversité voulus par la nature que de créer une forme d'homogénéité civique, et moins de proclamer une chimérique égalité abstraite que de donner corps, en mentionnant le fait que les droits sont inséparables de ses devoirs, à l'idée que le citoyen protégé par la loi est plus authentiquement libre - par la possession d'un statut juridique - que ne le serait l'homme de l'état de nature.

Le projet d'une religion civique enfin, même si cette " théophilanthropie » n'a jamais réellement pris corps, traduit l'inquiétude devant la capacité des désirs particuliers à subvertir le bien commun et à amoindrir le nécessaire amour de la patrie, ainsi que la nécessité de modeler les mœurs des individus en fonction des besoins de la république. Non seulement ce qui est privé continue d'être vu avec suspicion, mais les affections domestiques elles-mêmes sont tenues pour incapables de mener à une bienveillance sociale plus générale et soupçonnées d'engendrer des formes d'égoïsme et de partialité qu'il convient de maîtriser et de contenir. 
Andrew Jainchill en voit pour preuve la publication très officielle, sous le Directoire, d'un Manuel républicain de formation des mœurs et d'apprentissage des institutions qui témoigne de la prégnance du langage classique au même titre que, par exemple, l'Olbie de Jean-Baptiste Say où affleurent l'ensemble des anxiétés traditionnelles des républicains sur la manière dont le commerce et la propriété mobilière peuvent corrompre la pureté des mœurs et menacer la liberté en affaiblissant l'amour du bien public $^{19}$. Les mêmes préoccupations s'exprimaient au demeurant à propos de l'expansion extérieure et, sous le Directoire, l'idée selon laquelle c'est une telle politique qui avait perdu les républiques anciennes et tué l'amour de la liberté chez leurs citoyens, demeurait vivace.

Les thermidoriens ont donc conservé le langage de la république classique et leurs intuitions proto-libérales - nécessité de la stabilité, protection de la propriété - y demeurent comme emprisonnées. Le langage libéral affleurait cependant chez certains écrivains des lendemains de Thermidor. Charles Guillaume Théremin esquisse en particulier les contours d'une « république » libérale fondée sur un universalisme moral de type kantien ainsi que sur l'autonomie de la société civile, et attachée au développement d'une véritable civilisation marchande ${ }^{20}$. Théremin sait, bien avant Constant, que le monde moderne est essentiellement différent de l'Antiquité, que les hommes y cherchent non pas les vertus et les habitudes des républiques anciennes - dont les assises sont l'esclavage, la pauvreté privée et le sentiment d'abnégation envers la patrie - mais la prospérité matérielle et l'indépendance privée. Mais il ne va pas jusqu'à une célébration sans nuance du doux commerce et des mérites de la civilisation moderne car il remarque la propension des échanges internationaux à engendrer le conflit, ainsi que, plus généralement, les effets corrupteurs du commerce sur les mœurs d'un peuple libre. Il répugne aussi à prôner la substitution de la liberté civile à la liberté politique et conserve la thèse classique de la fécondité des conflits et de la nécessité de l'esprit civique. Jainchill repère les mêmes hésitations chez $\mathrm{M}^{\text {me }}$ de Staël : si celle-ci, dans son ouvrage Sur les circonstances actuelles qui peuvent terminer la révolution, fait l'apologie de la liberté comme indépendance et veut que chacun puisse conserver le loisir de demeurer à

(19) Andrew JaINChill, op. cit., p. 100-107; cf. Richard Whatmore, Republicanism and the French Revolution : An Intellectual History of Jean Baptiste Say's Political Economy, Oxford, Oxford University Press, 2000.

(20) Charles G. ThÉRemin, De l'incompatibilité du système démagogique avec le système d'économie politique des peuples modernes, Paris, chez les marchands de nouveautés, 1799. 
l'écart des affaires publiques, si elle prône le gouvernement représentatif et le renforcement de l'exécutif, l'importance qu'elle accorde aux mœurs, dit Jainchill, est « en tension avec sa conviction selon laquelle la république devrait refléter les intérêts des personnes privées » car elle conduit à réclamer «un certain degré d'homogénéité dans la population » et même à permettre « une orientation didactique des intérêts des citoyens $»^{21}$. Il y a bien là deux voix authentiquement libérales mais - selon Jainchill - elles furent peu entendues et, en tout état de cause, elles n'étaient pas entièrement exemptes de doutes sur la validité sans partage de la civilisation moderne et sur les mérites de l'indépendance individuelle. Jainchill rejoint donc Kalyvas et Katznelson pour affirmer que, même au lendemain de la Terreur, il n'y a guère de libéraux purs en France et que ceux qui ont conscience de la nécessité d'adapter le langage républicain aux impératifs d'une fondation politique stable savent aussi ce que le langage des droits individuels et de la société civile protégée contre l'État pourrait avoir de délétère et de dangereux pour l'esprit public et l'avenir de la liberté.

Paradoxalement, dit Jainchill, c'est dans le mouvement qui conduit à Brumaire que se sont forgés les premiers éléments d'un libéralisme français plus convaincu de sa propre validité. Les organisateurs du coup d'État -Sieyès entre autres - aspiraient en effet, à établir un régime moderne « fondé sur les vrais principes du gouvernement représentatif et sur les droits sacrés de la propriété, de l'égalité et de la liberté ». Leur modèle est, pourrait-on dire, antipolitique : ils veulent un État fort garantissant des libertés civiles, une répudiation de la démocratie au profit de la liberté privée, une politique réduite à l'administration des biens et à une organisation de la sûreté des personnes. Cet autoritarisme libéral, illustré par Pierre Louis Roederer autant que par Sieyès, sonne le glas du républicanisme classique chez les élites dirigeantes du Consulat. Roederer, par exemple, fait l'éloge sans phrases de la société commerciale et ne voit plus dans les peuples anciens que des barbares qui ignorent tout de l'art social, de la garantie des droits individuels, et de la manière de se procurer les jouissances matérielles et privées qui sont le seul fondement du bonheur humain ${ }^{22}$. Ici, enfin, la politique perd son rôle prééminent et devient la simple servante de la société civile et des intérêts individuels, de même que la liberté civile acquiert enfin la priorité qu'elle doit avoir sur une liberté politique qui, toujours portée à l'excès, finit toujours par l'op-

(21) Andrew JaINCHILL, op. cit., p. 133.

(22) Ibid., p. 206. 
primer. Si la démocratie menace la liberté, limitons-là sévèrement et faisons en sorte, comme le dit Sieyès, que l'autorité vienne d'en haut et que ce qui provienne du peuple ne soit plus que la confiance qu'il accorde à une autorité qui le protège ; ou encore, selon le mot de Cabanis, que l'on fasse tout pour le peuple, mais rien par le peuple.

Ce mouvement vers un autoritarisme libéral n'a cependant pas entraîné l'ensemble des esprits et la dérive du Consulat, puis de l'Empire, vers un exercice du pouvoir de moins en moins accueillant aux libertés civiles a fortement contribué à cette résistance. Mais, pour Jainchill, ces opposants - au nombre desquels Benjamin Constant occupe incontestablement la première place - se sont trouvés contraints par la situation de marier en quelque sorte leurs idées libérales à des éléments républicains : d'emblée, en effet, il apparaissait que c' était le projet même de confier le destin des libertés civiles à la seule garde d'un exécutif fort qui était erroné, car ces libertés personnelles ne peuvent avoir d'autre gardien que la liberté politique, et la société civile elle-même, loin de jouir d'une indépendance parfaite, ne peut avoir que la forme que lui donnent ses institutions politiques. Il n'est donc pas indifférent que ces dernières soient démocratiques car un régime autoritaire qui, par exemple, récuse l'indépendance de la justice, les garanties formelles et le droit d'appel, devient lui-même une entrave au développement d'une civilisation moderne et marchande. Au moment où il rédige Sur la possibilité d'une constitution républicaine pour un grand pays - entre 1801 et 1803 pour l'essentiel - Constant donne ainsi à ce qui va devenir la matrice du libéralisme français une tonalité spécifique issue de la constatation selon laquelle l'indépendance de la société par rapport à l'État ne suffit pas à garantir la liberté civile ${ }^{23}$ : comme il le dira encore en 1818 dans De la liberté des anciens comparée à celle des modernes, un ordre constitutionnel qui met la liberté au premier plan doit aussi pouvoir compter sur l'activité politique de ses citoyens pour faire échec au despotisme. De même si Constant multiplie, dans Sur la possibilité..., les mécanismes destinés à freiner ou à filtrer la démocratie de manière à assurer le pouvoir d'une élite de représentants éclairés, il n'en conclut pas moins que le système représentatif n'est qu'une « misérable parodie » sans le principe électif et qu'un exécutif unique et concentré constitue toujours pour la liberté un ennemi potentiellement dangereux.

(23) Ibid., p. 275. 
Daunou, Lanjuinais, Sismondi et surtout Tocqueville emboîteront le pas à Constant pour souligner que la politique et les institutions libres sont les remparts de la liberté individuelle. C'est bien l'auteur de la Démocratie en Amérique qui explique comment les Américains ont utilisé la liberté - politique ! - pour contenir la menace que la privatisation, l'individualisme et l'appétit des jouissances font peser sur les libertés personnelles et combler l'espace que ces maux de l'époque moderne offrent au développement d'une forme nouvelle de despotisme : «Une nation qui ne demande rien de plus à son gouvernement que le maintien de l'ordre, écrit-il, est déjà esclave au fond de son cœur $»^{24}$.

Constant et Tocqueville, conclut Andrew Jainchill, «partagent la conviction que la liberté des modernes est insuffisante et qu'elle doit être contrebalancée par un solide attachement à la liberté des anciens ». Cette conviction, dit-il, fait ressortir le caractère distinctif du libéralisme français, fortement teinté de républicanisme par ses origines : non seulement il croit au caractère incontournable de la liberté politique pour contenir les dérives jumelles de l'individualisme et de l'autoritarisme, mais il croit aussi que cette activité politique est une dimension irremplaçable de l'existence humaine et que les institutions politiques sont largement fondées à en inculquer la valeur aux citoyens, pratiquant ainsi une forme de perfectionnisme qui aura toujours été étranger aux libéraux anglosaxons.

Comme on va tenter de le montrer, ces deux analyses de la naissance du langage libéral et du rapport de répudiation et d'enveloppement qu'il entretiendrait avec son prédécesseur républicain ne sont pas entièrement satisfaisantes.

Tout d'abord, l'idée d'une généalogie unique est certainement valide, même si Kalyvas et Katznelson demeurent dans le flou lorsqu'il s'agit de préciser d'où viennent les éléments extérieurs qui ont été importés dans le langage républicain et qui ont largement présidé à son adaptation, principalement la conception des droits naturels et l'idée de pluralisme moral et religieux. S'agit-il d'éléments épars et qu'il serait impossible de définir comme faisant partie d'un langage politique déjà constitué comme « libéral », comme ils le prétendent, ou bien s'agit-il au contraire de parties composantes d'un langage constitué qui se présente comme une alternative complète au républicanisme classique ? Certes, on sait que la théorie de la dualité des vocabulaires proposée par J.G.A. 
Pocock se heurte au fait que le langage des droits et celui de la vertu n'étaient pas perçus au XVIII ${ }^{\mathrm{e}}$ siècle comme des langages opposés ou alternatifs et qu'ils étaient maniés de manière conjointe et indifférenciée par l'ensemble des opposants à la monarchie sans que jamais ceux-ci voient la moindre incohérence dans la tactique consistant à emprunter simultanément des arguments au vocabulaire classique de la vertu et au vocabulaire moderne des droits et du contrat. Mais la question mériterait au moins d'être abordée.

En second lieu, l'analyse de la manière dont le langage républicain s'est dépouillé de ses aspects « archaïques » pour s'adapter aux exigences de la société moderne ( vertu, participation civique exacerbée, homogénéité, patriotisme, agonistique politique, militarisme, patriotisme) est également indiscutable, mais le problème est qu'aucun de ces éléments n'est partie intégrante de l'analyse de ce que les républicains d'aujourd'hui présentent comme l'élément central de la théorie politique républicaine, à savoir le concept de liberté comme non-domination. Au contraire, non seulement ce concept est compatible avec le rejet de ces éléments mais il paraît même l'exiger. C'est le cas de tout ce qui, dans le langage républicain « classique », porte la marque de la cité ancienne : thème du noble loisir citoyen par opposition aux activités serviles de la production et de l'échange, vertu, caractère central de la participation civique dans la définition de la nature humaine, monolithisme moral, communauté fermée, refus de l'égalité entre les sexes, théorie de l'élite civique, patriotisme étroit, militarisme, etc. En revanche, l'un des éléments constitutifs de la théorie républicaine classique, à savoir le souci des conditions matérielles de l'indépendance du citoyen, est coextensif à l'idée de liberté comme non-domination. Comme nombre d'auteurs l'ont souligné entre 1750 et 1850 - et au-delà - la liberté en ce sens républicain n'est pas compatible avec une théorie des droits qui ferait du droit d'appropriation un droit illimité et absolu, dans la mesure où l'exercice d'un tel « droit » aboutit à des formes d'inégalité matérielle où l'indépendance et l'accès aux conditions de la non-domination ne sont plus garantis pour l'ensemble des citoyens ${ }^{25}$. La question centrale - qui n'est jamais posée dans ces deux ouvrages alors même que Katznelson et Kalyvas évoquent par exemple la tension entre le marché et la souveraineté populaire comme l'un des

(25) Cf. par exemple Destutt de Tracy, Traité de la volonté et de ses effets (1818), rééd. Paris, Fayard, 1994, p. 214-215. Comme le montrent Kalyvas et Katznelson, Adam Ferguson avait aussi développé cette position dans son Histoire de la société civile avant de la répudier dans ses travaux ultérieurs. 
dilemmes centraux qui se posent au langage du libéralisme naissant - est donc bien celle-ci : le droit de s'approprier sans limite les ressources de la nature est-il un droit inhérent à l'individu, un droit qui pourrait s'exercer sans aucune préoccupation pour la manière dont cet exercice affecte la possibilité que les autres ont d'accéder aux conditions de la non-domination $^{26}$ ? La garantie d'un tel droit est-elle indispensable à la cohérence du langage politique libéral ? Parmi les évolutions et les adaptations subies par le langage républicain au moment où il était appelé à occuper la place laissée vide par l'effondrement du despotisme monarchique, est-ce que l'on repère explicitement cette détermination à abandonner complètement l'idée que l'économie et la chrématistique doivent être subordonnées à l'impératif de non-domination et par conséquent assujetties à un certain impératif d'égalité matérielle destiné à éviter la conversion des inégalités de conditions en inégalités de pouvoir? Il ne fait aucun doute que certains ont explicitement voulu cette adaptation en pensant que la société devait se réorienter vers l'objectif majeur de la production des biens et que, admettant le droit absolu d'appropriation au nombre des droits naturels, ils ont par là accepté cette révolution des rapports de subordination entre l'économique et le politique qui a fait d'eux des libéraux. Cette conclusion est partagée par tous ceux qui sont convaincus que le droit de propriété est coextensif à la civilisation, indispensable à la distinction décisive entre la société civile et l'État et, au-delà, à la liberté des individus comme non intervention de l'autorité collective dans la vie des individus. Tous ceux qui se sont convaincus qu'il existait un lien intrinsèque entre les droits civils personnels (liberté de conscience, de presse, de pensée, de choix de l'occupation etc.) et le droit de propriété ont donc affirmé que toute tentative pour limiter ce dernier au nom de l'égalité dans les conditions d'accès à l'indépendance était définitionnellement anti-moderne et qu'elle aboutissait nécessairement, sous le poids de l'intervention de l'autorité collective au nom de l'égalité, à la mort de l'indépendance individuelle qu'elle prétendait garantir. Ceux-là sont à l'évidence devenus des libéraux. Andrew Jainchill a sans doute raison de souligner que, au lendemain de la Révolution, il s'est trouvé fort peu d'esprits pour se convertir sans phrase à l'idée que la société civile devait consister en une sphère d'activités exclusivement privées pour laquelle l'exercice actif des droits politiques pourrait représenter une menace, mais cela cesse d'être le cas après 1815 . Il a par ailleurs certainement tort de laisser penser que l'attachement à la liberté politique est le seul

(26) $C f$. la réponse négative de Paine dans Agrarian Justice. 
signe tangible de la survie du langage républicain après Thermidor car, audelà, la question de l'inégalité, de l'ancrage de la personnalité civique dans les moyens de l'indépendance, et au fond de la possibilité de la liberté individuelle dans une société livrée à la domination sans partage du droit de propriété reste posée, d'abord chez Babeuf et Buonarroti, puis chez les républicains « socialistes » de la première moitié du XIX ${ }^{\mathrm{e}}$ siècle ${ }^{27}$.

En ce sens, prétendre que le langage républicain a disparu en tant qu'option alternative à un ensemble d'affirmations proto-libérales, ou qu'il n'existe plus qu'à l'état de reste dans un langage entièrement différent, c'est prétendre qu'il ne s'est trouvé personne pour penser que, dans les conditions modernes, le souci de l'indépendance personnelle de chacun exigeait une réflexion sur les bases matérielles de celle-ci et pour en conclure que, dans une société d'individus pourvus de droits, l'impératif de liberté dit en termes de non-domination exigeait un refus des monopoles, un contrôle des inégalités et des mécanismes de translation du pouvoir économique en pouvoir politique, ainsi qu'une production publique et collective de dispositifs institutionnels permettant aux non propriétaires ou aux plus démunis d'échapper à la dépendance et à la nécessité de la déférence envers ceux qui sont maîtres de leurs conditions matérielles d'existence. Ces dispositifs n'incluent pas seulement des droits politiques mais aussi l'ensemble des droits sociaux promus par l'État qui rétablissent, pour les moins bien armés par la nature ou par l'héritage, des moyens de négociation que les simples mécanismes marchands ne leur fournissent pas. Ceux qui ont été convaincus que cette limitation de la liberté d'appropriation au nom de liberté de choix était une condition de la légitimité dans les sociétés d'individus sont demeurés des républicains. Pas au sens classique - car ils ne croient pas à la vertu ni à la communauté fermée - mais au sens moderne, qui leur permet de définir la république comme une forme de société soucieuse de rétablir la légitimité des rapports individuels de compétition en rétablissant une certaine égalité dans ce que Durkheim appelle « les conditions extérieures de la lutte ». Ils sont convaincus que l'exercice des droits politiques par l'ensemble des membres de la communauté civique est la condition préalable de la réalisation des conditions d'une telle égalité mais ils sont aussi convaincus que la démocratie n'est qu'un moyen d'un objectif que Louis Blanc appellera « la régénération sociale ${ }^{28}$.

(27) Cf. par exemple les articles de Louis Blanc dans les premiers numéros de la Revue du Progrès, politique, social et littéraire ( $\mathrm{n}^{\circ} 1$ et 2,1839$)$.

(28) Louis Blanc, "La république, le moyen, la régénération sociale, le but », dans Questions, d'aujourd'hui et de demain, Paris, Dentu, 1873-1880, t. 5, p. 455-460. 
C'est bien la raison pour laquelle la dimension analytique demeure incontournable. Il faut poser la question de la définition d'une « république », c'est-à-dire d'un régime institutionnel et social qui apparaisse non comme un instrument d'assujettissement de certains individus à certains autres, mais comme un instrument général de libération et d'autonomisation. En un moment social où les liens communautaires fondés sur des croyances partagées ont largement laissé la place à un extraordinaire pluralisme des convictions et des modes de vie, le fondement de la légitimité des institutions ne peut en effet plus se trouver que dans la justice, c'est-à-dire dans une forme de régulation sociale qui garantit à chacun qu'il n'est pas l'instrument de l'indépendance des autres et qu'il possède autant de chances que quiconque de donner corps à ses propres projets. En son acception moderne, la théorie politique républicaine est donc une théorie de la légitimité de l'autorité et des règles collectives dans une société d'individus séparés ; elle affirme que, dans un contexte de ce genre, les individus ne sont libres que si les règles sont légitimes et que, à leur tour, les règles ne sont légitimes que si elles peuvent rationnellement être considérées par tous non comme des outils de pouvoir mais comme des instruments d'affranchissement. Cette exigence signifie que les règles en question doivent s'employer activement à rétablir, avec une garantie publique, une certaine forme de justice et d'équilibre des pouvoirs dans les relations de personne à personne qui est la condition de la non-domination. Cet impératif-là n'est pas mort. On a certes raison de dire qu'il n'exige pas une imitation des modèles antiques et que, à ce titre, une bonne part du langage républicain tel que la tradition l'a transmis à l'époque moderne est définitivement obsolète: la vertu n'a jamais donné lieu qu'à l'oppression du grand nombre par l'élite et le thème de la participation civique, toujours fantasmé dans les conditions modernes, ouvre la voie à toutes les répressions. Mais la mort de ce langage dépassé - et incapable de contribuer utilement à une gouvernance stable et légitime - signifie-t-elle que l'on doit jeter en même temps par-dessus bord le cœur de la théorie républicaine classique à savoir l'impératif d'égalité dans l'accès à la liberté ? Certainement pas. Quant à la teinte spécifique du libéralisme français que Jainchill prétend isoler - il serait demeuré sensible à l'impératif de la participation politique au lieu de tout sacrifier au dogme de l'indépendance privée - il a surtout pour effet de dissimuler l'essentiel : l'attachement que la culture politique française a su préserver pour la justice et l'égalité comme fondements d'une authentique liberté moderne et le refus 
persistant d'accepter l'idée que le déploiement des droits individuels doive nécessairement se payer de la prolifération des dominations privées.

Jean-Fabien SpITZ

UFR de philosophie Université de Paris I Panthéon Sorbonne / Institut universitaire de France 17, rue de la Sorbonne 75005 Paris Institut Universitaire de France jfspitz@univ-paris1.fr 
